\title{
Electro-optic delay devices with double feedback
}

\author{
Romain Modeste Nguimdo, Pere Colet, and Claudio Mirasso
}

\begin{abstract}
We analytically and numerically study the effect of an additional feedback in the semiconductor laser used to pump optoelectronic delay devices. We show that this additional feedback renders the system into chaotic regime for a broader parameter range and also induces a stronger chaotic behavior. We study the synchronization of this system as function of the parameter mismatch and show its capability for encoded message transmission.
\end{abstract}

Index Terms-Chaos based communications, Chaos generation, Synchronization, Opto-electronic feedback

\section{INTRODUCTION}

Devices with opto-electronic feedback loops show a rich variety of dynamical behaviors. In particular, they have been used as chaos generator for chaos-based communications [1], [2]. Typically the devices used for that aim consist of an interferometer fed by a $\mathrm{CW}$ semiconductor laser plus a delay loop which modifies the optical length of one of the interferometer arms. The interplay between nonlinearity and delay generates a chaotic output carrier in which a message can be encoded. Recovery of the message is based on the fact that, under appropriate conditions, similar chaotic system can synchronize with the emitter [3]. The receiver system replicates only the chaotic carrier and by comparing the input of the receiver (the chaotic carrier with message) with the output (the chaotic carrier) one can extract the message as originally shown in electrical circuits [4]. In optical systems there were early proposals for solid state lasers [5] and semiconductor lasers [6], [7]. Experimental demonstrations came at late '90 using fiber lasers [8] and optoelectronic feedback devices [1], [2]. The field has grown and an experiment in the optical network of Athens has paved the road towards practical applications [9]. Several schemes have been used to induce chaos in semiconductor lasers including all-optical feedback or optoelectronic feedback [10].

Optical chaos encrypted communications require wide spectrum of the chaotic carrier to efficiently mask the message from eventual eavesdroppers. This goal can be achieved in optoelectronic devices by increasing the gain loop parameter [11], [12]. However, experimental observations have shown that the gain loop is limited due to the saturation introduced by the bandwidth of the device components. Since then, it becomes necessary to investigate an alternative way to produce a stronger chaos. Moreover, the increase in the number of the

Authors are with the Instituto de Física Interdisciplinar y Sistemas Complejos, IFISC (CSIC-UIB), Campus Universitat de les Illes Balears, E-07122 Palma de Mallorca, SPAIN

We gratefully thank Vasile Tronciu for helping us with the DDE-BIFTOOL program. As well, financial support from MICINN, Spain, and Feder under Project TEC2006-1009/MIC (PhoDECC) and Project FIS2004-00953 (CONOCE2) and by the EC Project PICASSO (IST-2005-34551) is acknowledged. R.M.Nguimdo also acknoledges the fellowship BES-2007-14627 under the FPI program of MICINN (Spanish Goverment). system parameters can also be fruitful to provide more security since an eavesdropper has to face up the parameter mismatches to succeed in building an adequate receiver necessary for the decryption of the message. A way to reach all these purposes can consist in evolving an extra feedback loop feeding the laser. It is worth noting that the feedback in the controlled source has previously considered in opto-electronic systems by Udaltsov and al. as a possible way to get around timedelay cracking [13].

In this paper, we explore the impact of such combination taking the inspiration from the existing model exhibited in [1]. The work is organized as follows: In section II, we present two new proposals differing in the way the laser feedback is performed. Section III is devoted to the theoretical and numerical results of each model while section IV deals with the synchronization and the effects of parameter mismatch. In section $\mathrm{V}$, the viability of the models is numerically tested by encoding and decoding the message. Section VI highlights some concluding remarks and outlook.

\section{THE SYSTEM}
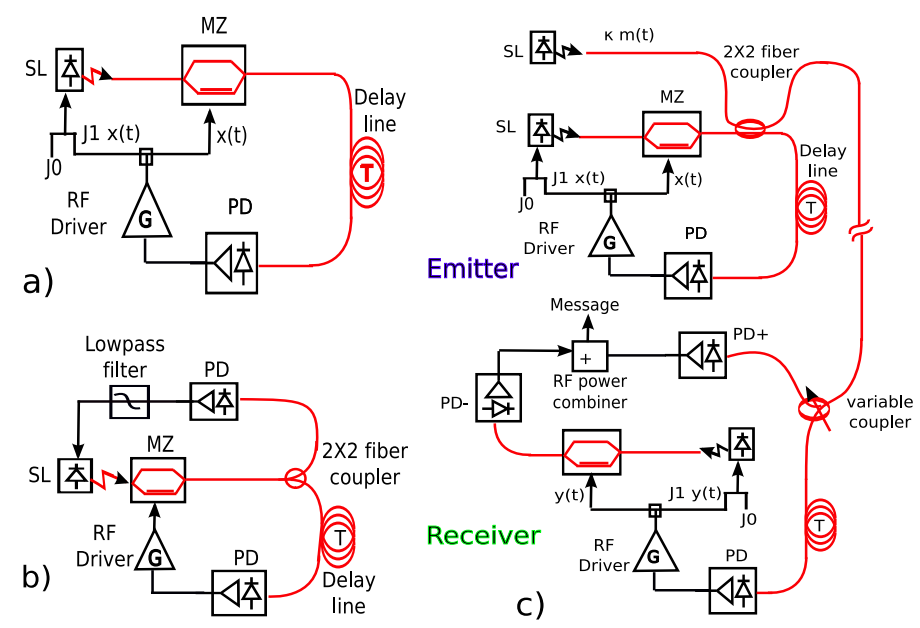

Fig. 1. Schematic setup: a) Emitter for System I; b) Emitter for System II; c) Full emitter-receiver setup for system I.

We consider two systems for chaos generation whose schematic setups are shown in Figs. 1 a) (system I) and 1 b) (system II). Each emitter is composed of:

- A semiconductor laser (SL) delivering a power $P=h \nu I$ where $h$ stands for the Planck constant, $\nu$ is the photon emission frequency and $I$ the photon number.

- A Mach-Zehnder (MZ) modulator: The light coming from the SL is equally split into the two arms of the $\mathrm{MZ}$ and interferes at its output because of the one arm refractive index variation. Effectively, this arm is 
modulated by the output voltage of an electronic driver. The applied voltage has two components: a constant or DC component $V_{B}$ that allows to select the operating point of the modulator; and a radio-frequency (RF) component $V(t)$ which is used to generate the chaos. Considering an optical input $P$, the $\mathrm{MZ}$ output is written as $P \cos ^{2}\left[\pi V(t) /\left(2 V_{\pi R F}\right)+\pi V_{B} /\left(2 V_{\pi D C}\right)\right]$ where $V_{\pi R F}$ and $V_{\pi D c}$ stand for the RF half-wave and the bias electrode half-wave, respectively.

- A fiber delay line used to delay the optical signal in time.

- An amplified diode with sensitivity $s$ to detect the optical signal and convert it into an electrical signal.

- A RF driver whose output modulates the MZ and closes the delay loop.

For the modeling purposes, we introduce $x(t)=$ $\pi V(t) /\left(2 V_{\pi R F}\right)$ and $w=\int_{t_{0}}^{t} x\left(t^{\prime}\right) d t^{\prime}$. Assuming that the RF amplifier behaves as a second-order bandpass linear filter with gain $G$ the system can be described by

$$
\begin{aligned}
x+\tau \frac{d x}{d t}+\frac{1}{\theta} w & =\beta_{1} I(t-T) \cos ^{2}[x(t-T)+\phi], \\
\frac{d w}{d t} & =x,
\end{aligned}
$$

where the parameters are the fast cutoff time-scale $\tau$, the slow cutoff response time $\theta$, the offset phase $\phi=\pi V_{B} /\left(2 V_{\pi_{D C}}\right)$, delay time $T$ and the normalized electro-optical gain coefficient $\beta_{1}=\pi s G k / 2 V_{\pi R F}$, where $k$ accounts for overall losses. The photon number $I$ obeys the laser rate equations [14]:

$$
\begin{aligned}
\frac{d I}{d t} & =(\mathcal{G}-\gamma) I \\
\frac{d N}{d t} & =J_{0}-\gamma_{e} N-\mathcal{G} I+F(x) \\
\mathcal{G} & =g\left(N-N_{0}\right) /(1+S I),
\end{aligned}
$$

where $N$ is the carrier number, $g$ is the differential gain parameter, $S$ is the gain saturation factor, $N_{0}$ is the carrier number at transparency, $\gamma$ is the inverse photon lifetime, $\gamma_{e}$ is the inverse carrier lifetime. The gain saturation factor given by $(1+S I)$ is included in [15] to summarize a set of physical effects that eventually bound the material gain as the number of intracavity photons increases. We consider that the injected current has a DC component $J_{0}$ and a feedback contribution $F(x)$ which is a function of the RF voltage as follows:

System I: A fraction $J_{1}$ of the RF driver output is injected back to the laser, such that

$$
F(x)=J_{1} x(t) .
$$

Since $x(t)$ is a dimensionless variable, $J_{1}$ has the same unit as $J_{0}$.

System II: Using $2 \times 2$ fiber coupler and an additional photodetector, a fraction of the optical light is detected at the output of $\mathrm{MZ}$ and converted into electrical signal. In this process those frequencies larger than the photodetector bandwidth $f_{c}$ are filtered out, we model this as a lowpass filter having input signal $J_{1} \beta_{1} I \cos ^{2}[x(t)+\phi]$ which comes from converting the optical signal into an electric one. The feedback contribution in this case is then given by

$$
\frac{d F}{d t}=-2 \pi f_{c}\left\{F-J_{1} \beta_{1} I \cos ^{2}[x(t)+\phi]\right\} .
$$

In the absence of feedback to the pump current, the laser delivers a constant power and the overall electro-optical gain $\beta=\beta_{1} I$ is thereby constant. However, with the extrafeedback, the photon number $I$ switches over a regime implicitly depending on the RF voltage.

We note that the presence of the low cutoff integral term $w(t)$ in Eq. (1) imposes the mean value $\langle x(t)\rangle$ to be zero in order to ensure the convergence of the solution even at infinite time. This is true for the case of no feedback in the laser as well as for the two new systems introduced here. For system I the average of the feedback dependent current injected in the laser $F$ has a zero average, so that the average of the output intensity $\langle I\rangle$ is the same as the constant laser power without the feedback, and $\beta_{1}\langle I\rangle=\beta$. That is not the case for system II.

\section{THEORETICAL AND NUMERICAL STUDY}

For the numerical study, we take the following parameter values $g=1.5 \times 10^{-8} \mathrm{ps}^{-1}, S=2 \times 10^{-7}, N_{0}=1.2 \times 10^{8}$, $\gamma=3.3 \times 10^{11} \mathrm{~s}^{-1}, \gamma_{e}=5 \times 10^{8} \mathrm{~s}^{-1}$ [16]. With these parameters, the threshold current is $J_{t h}=7.1 \times 10^{16} \mathrm{~s}^{-1}$ (corresponding to a threshold current intensity of $11.8 \mathrm{~mA}$ ). Also, we consider $\tau=25 \mathrm{ps}, \theta=5 \mu \mathrm{s}, \beta_{1}=2.89 \times 10^{-5}$, $T=2.5 \mathrm{~ns}$. Others parameters will be stated in the figure captions.

\section{A. System I}

When $d x / d t=d w / d t=0$, there is a fixed point solution $\left(x_{s t}, w_{s t}, I_{s t}, N_{s t}\right)$ where

$$
\begin{aligned}
x_{s t} & =0, \\
w_{s t} & =\theta \beta_{1} I_{s t} \cos ^{2} \phi \\
I_{s t} & =\frac{J_{0} g-\gamma_{e} g N_{0}-\gamma_{e} \gamma}{\gamma\left(g+S \gamma_{e}\right)}, \\
N_{s t} & =\frac{g N_{0}+\gamma+S J_{0}}{g+S \gamma_{e}} .
\end{aligned}
$$

Assuming $x=x_{s t}+\delta x, w=w_{s t}+\delta w, I=I_{s t}+\delta I$ and $N=N_{s t}+\delta N$, and linearizing Eq. (1) around the fixed point, the stability of this single fixed point is determined by the following $4 \times 4$ matrix

$$
M=\left(\begin{array}{cccc}
\tilde{a}_{11}+a_{11} e^{-\lambda T} & a_{12} & a_{13} e^{-\lambda T} & 0 \\
1 & 0 & 0 & 0 \\
0 & 0 & a_{33} & a_{34} \\
J_{1} & 0 & a_{43} & a_{44}
\end{array}\right)
$$

where the coefficients are

$$
\begin{aligned}
& a_{11}=-\frac{\beta_{1} I_{s t}}{\tau} \sin 2 \phi, \tilde{a}_{11}=-\frac{1}{\tau}, a_{12}=-\frac{1}{\tau \theta}, \\
& a_{13}=\frac{\beta_{1} \cos ^{2} \phi}{\tau}, a_{33}=-\frac{S \gamma I_{s t}}{1+S I_{s t}}, a_{34}=\frac{g I_{s t}}{1+S I_{s t}}, \\
& a_{41}=J_{1}, a_{43}=\frac{-\gamma}{1+S I_{s t}}, a_{44}=-\gamma_{e}-\frac{g I_{s t}}{1+S I_{s t}} .
\end{aligned}
$$

The steady state is stable if the real part of all the eigenvalues $\lambda$ are negative. The spectral analysis of delayed systems is more involved than that of ordinary systems since the term $e^{-\lambda T}$ in the matrix $M$ leads to a characteristic equation which can have an infinite number of eigenvalues. Out of all those eigenvalues, 

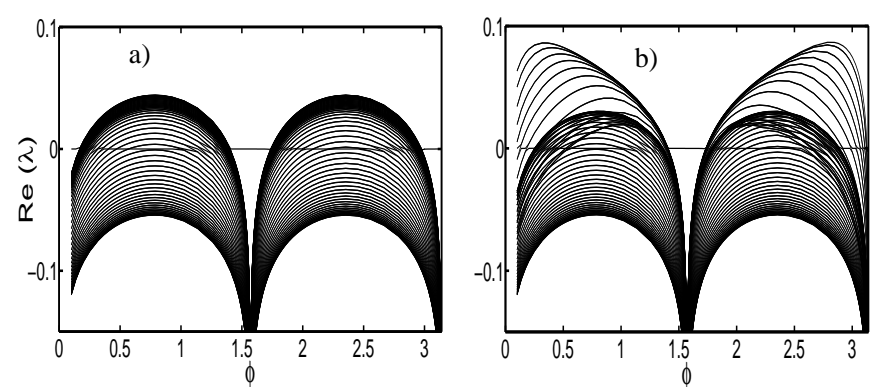

Fig. 2. Real part of the top $\mathrm{N}$ eigenvalues versus offset phase for $J_{1}=0.0$ and $J_{1}=0.1 J_{0}$. We have chosen $J_{0}=1.5 J_{t h}$ which corresponds to $\langle\beta\rangle=$ $\beta_{1}\langle I\rangle=3.08$.
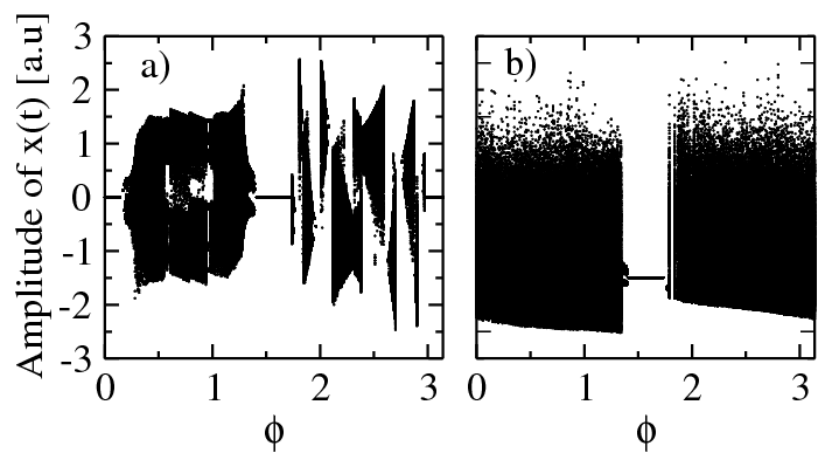

Fig. 3. Bifurcation diagram versus offset phase $\phi$ for $J_{0}=1.5 J_{t h}$ : a) $J_{1}=0.0$ and b) $J_{1}=0.1 J_{0}$

typically one computes a finite set of the $N$ eigenvalues with largest real part. One of the packages that can be used for this purpose involves the DDE-BIFTOOL algorithm in matlab [17]. In this method characteristic roots are computed through successive approximations. We henceforth use this method to investigate the stability of the steady state given above. The presence of $\sin 2 \phi$ and $\cos ^{2} \phi$ in the matrix coefficients shows that the function is $\pi$-periodic. Therefore we can perform the stability analysis only over the interval $[0, \pi]$ without loosing generality. Figure 2 shows the real part of the top $N$ eigenvalues of $M$. When the feedback is only applied to the $\mathrm{MZ}\left(J_{1}=0\right)($ Fig. $\left.2 \mathrm{a})\right)$, there are three stable regions (around zero, $\pi / 2$ and $\pi$ ) corresponding to the offset phases which lead to the predominance of destructive interference. Adding the feedback to the SL the stationary state for $\phi=0$ or $\pi$ is destabilized while the stationary state corresponding to $\phi=\pi / 2$ remains stable (see Fig. 2 b) for $J_{1}=0.1 J_{0}$ ).

Figure 3 a) shows the bifurcation diagram for $J_{1}=0$ displaying all the maxima and minima of a long time trace obtained for each value of the offset phase $\phi$. All the results are obtained integrating over a time of $80 \mu \mathrm{s}$ which is 16 times longer than the slowest time scale $\theta$ of the model. The time step used for the numerical integration is $1 \mathrm{ps}$. The single value for the amplitude maxima found for phases close to zero, $\pi / 2$ and $\pi$ corresponds to the stable steady state described in the previous paragraph. Multiple values for the amplitude maxima or minima correspond to multiperiodic or chaotic behaviors. Figure $3 \mathrm{~b}$ ) shows the corresponding bifurcation diagram for $J_{1}=0.1 J_{0}$. As predicted by the linear stability analysis, the
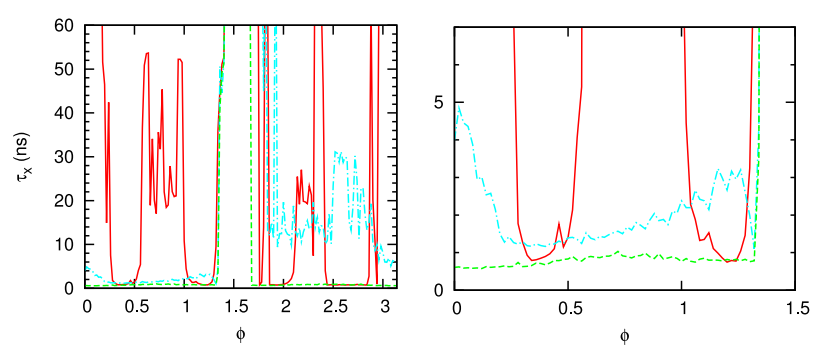

Fig. 4. Auto-correlation time versus offset phase $\phi$ for $J_{1}=0.0$ (solid line), $J_{1}=0.1 J_{0}$ for system I (dotdashed line) and $J_{1}=0.1 J_{0}$ for system II (dot line). The right panel represents the zoom of the left panel. $\tau_{x}>60 \mathrm{~ns}$ corresponds to the steady state in our case.

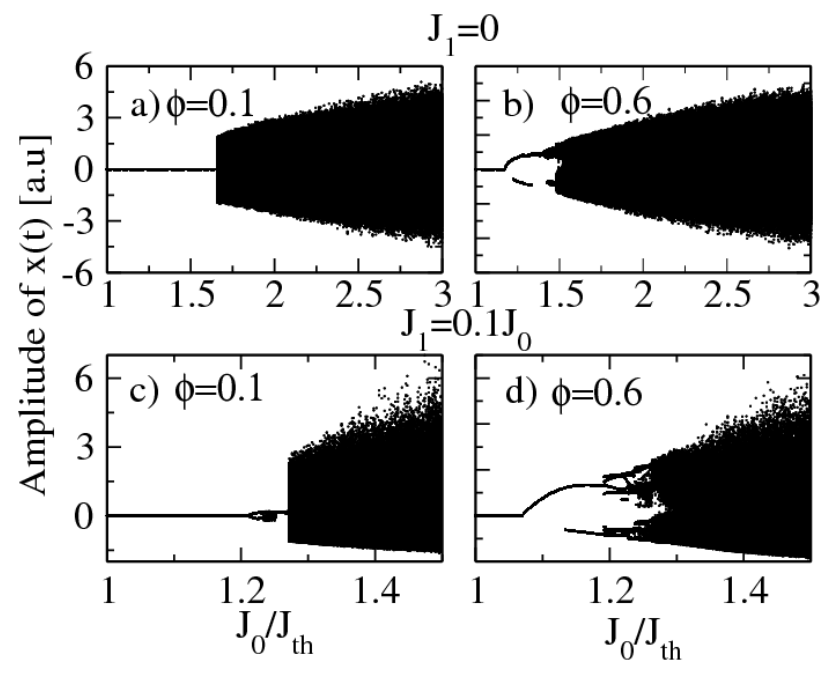

Fig. 5. Bifurcation diagram versus the injection current $J_{0}$ for offset phases $\phi=0.1$ (left panels) and $\phi=0.6$ (right panels). We have taken $J_{1}=0.0$ (top row) and $J_{1}=0.1 J_{0}$ (bottom row)

steady state solutions for off-set phase $\phi=0$ and $\phi=\pi$ are now unstable and the system displays a chaotic behaviors. Furthermore inspecting the values of the offset phases for which the system is already unstable, we see that typically the spread of bifurcation diagram is larger and denser indicating that the system has become more chaotic by applying feedback in the laser.

Quantitatively speaking, we can efficiently characterize the chaos by calculating the autocorrelation time defined as:

$$
\tau_{x}=\int_{0}^{\infty} \Gamma_{x x}\left(t^{\prime}\right) d t^{\prime}
$$

where $\Gamma_{x x}$ stands for the normalized auto-correlation function defined as

$$
\Gamma_{x x}\left(t^{\prime}\right)=\frac{\left\langle[x(t)-\langle x(t)\rangle]\left[x\left(t+t^{\prime}\right)-\langle x(t)\rangle\right]\right\rangle}{[\langle x(t)-\langle x(t)\rangle\rangle]^{2}} .
$$

Figure 4 displays the correlation time $\tau_{x}$ as a function of $\phi$. It can be clearly seen that for most values of $\phi$ the system becomes more chaotic (smaller correlation time) by applying the feedback in the laser. Also, there are some values of $\phi$ for which the system is in fact less chaotic. 

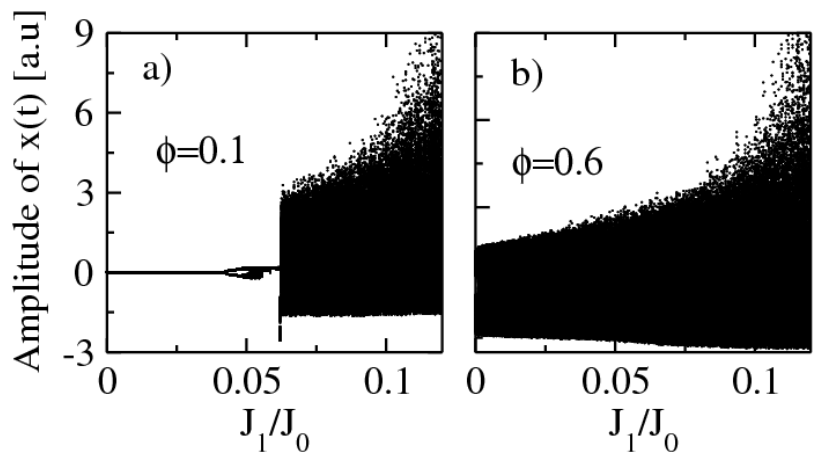

Fig. 6. Bifurcation diagram versus $J_{1}$ for $J_{0}=1.5 J_{t h}$ : a) $\phi=0.1$ and b) $\phi=0.6$

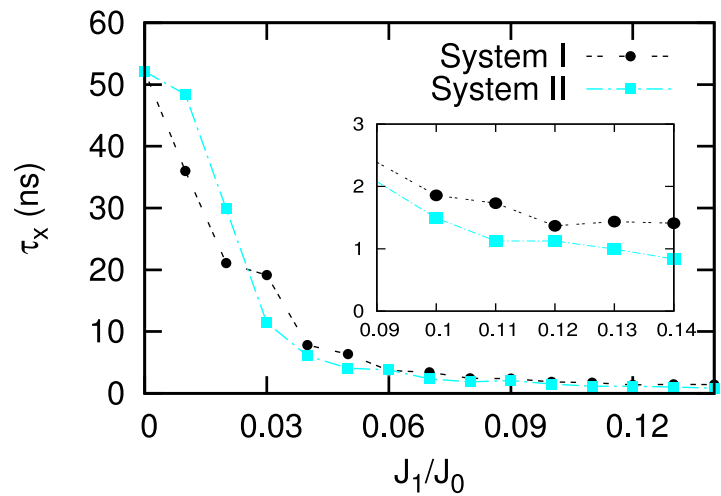

Fig. 7. Auto-correlation time with respect to $J_{1}$ for $J_{0}=1.5 J_{t h}$ and $\phi=0.6$ considering system I and system II. The inset shows the zoom plot when $\tau_{x}$ decreases enough.

Different dynamical regimes can be observed by simply changing the pump current $J_{0}$. Figures 5 a) and b) display the bifurcation diagram as function of $J_{0}$ when there is no feedback in the laser for two values of the offset phase. Depending on the off-set phase some periodic windows are observed while chaotic behaviors appear typically beyond $J_{0} \gtrsim 1.6 J_{t h}$. When adding the feedback to the laser $\left(J_{1}=0.1 J_{0}\right)$ the chaotic behavior develops earlier for $J_{0} \gtrsim 1.3 J_{t h}$ and is stronger as can be seen in Fig. $5 \mathrm{c}$ ) and d).

Figure 6 shows the bifurcation diagram as function of $J_{1}$ while keeping constant $J_{0}=1.5 J_{t h}$ for the same offset phases as before. In Fig. 6 a), it appears some threshold $\left(J_{1} \approx 0.06 J_{0}\right)$ of $J_{1}$ for which the system shows chaotic behavior. For $\phi=0.6$ (Fig. 6 b)) the system is already chaotic with no feedback in the laser. The bifurcation diagram indicates that the dispersion of the maxima of the time trace is larger increasing $J_{1}$. A more quantitative characterization can be done using again the correlation time. Figure 7 shows the autocorrelation time $\tau_{x}$ as function of $J_{1}$ for a fix value of $J_{0}$ and offset phase $\phi=0.6$. The decrease in the autocorrelation time indicates that the system becomes more chaotic as $J_{1}$ increases up to $J_{1} \approx 0.12 J_{0}$. After that the autocorrelation time slightly increases so that increasing $J_{1}$ beyond that level does not provide a more chaotic behavior (see inset).
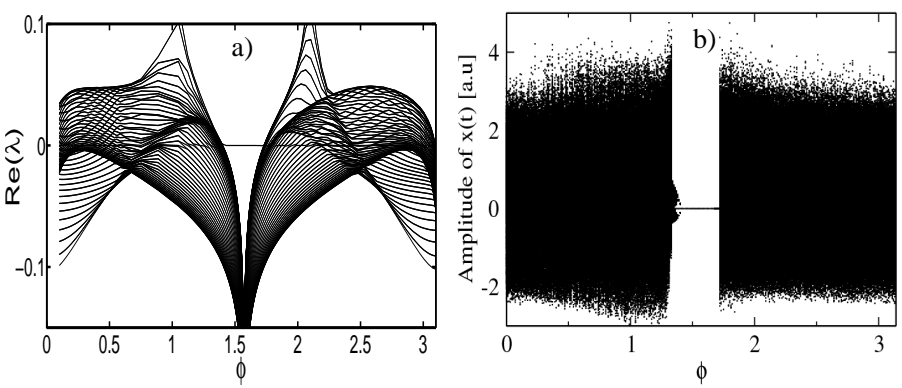

Fig. 8. a) Real part of the top $\mathrm{N}$ eigenvalues of matrix $M$ and b) bifurcation diagram versus off-set phase for $J_{0}=1.5 J_{t h}$ and $J_{1}=0.1 J_{0}$

\section{B. System II}

The fixed point of this model is given by $\left(x_{s t}, w_{s t}, I_{s t}, N_{s t}, F_{s t}\right)$ where

$$
\begin{aligned}
& x_{s t}=0, \\
& w_{s t}=\theta \beta_{1} I_{s} \cos ^{2} \phi, \\
& I_{s t}=\frac{\left(J_{0}+F_{s}\right) g-\gamma_{e} g N_{0}-\gamma_{e} \gamma}{\gamma\left(g+S \gamma_{e}\right)}, \\
& N_{s t}=\frac{g N_{0}+\gamma+S\left(J_{0}+F_{s}\right)}{g+S \gamma_{e}}, \\
& F_{s t}=J_{1} \beta_{1} I_{s t} \cos ^{2} \phi .
\end{aligned}
$$

We should note that for this system the photon number at the steady state is larger and depends on the offset phase. The stability of this fixed point can be investigated following an approach similar to that of subsection III-A. Since now we have an extra equation (Eq. (7)) we have to deal with the $5 \times 5$ matrix

$$
M=\left(\begin{array}{ccccc}
\tilde{a}_{11}+a_{11} e^{-\lambda T} & a_{12} & a_{13} e^{-\lambda T} & 0 & 0 \\
1 & 0 & 0 & 0 & 0 \\
0 & 0 & a_{33} & a_{34} & 0 \\
0 & 0 & a_{43} & a_{44} & 0 \\
\eta I_{s t} \sin (2 \phi) & 0 & -\eta \cos ^{2} \phi & 0 & -2 \pi f_{c}
\end{array}\right)
$$

where $\eta=2 \pi f_{c} J_{1} \beta_{1}$ and the coefficients $a_{i j}$ are given by Eq. (9) with $I_{s t}$ given by Eq. (12). The real part of the top $N$ eigenvalues of this matrix are plotted in Fig. 8. As happens for system I the feedback in the laser destabilizes the steady state around the offset phases 0 and $\pi$ while a narrow region of stability for the stationary state remains for an offset phase around $\phi=\pi / 2$. Figure 8 b) shows the bifurcation diagram as a function of the offset phase. Comparing this figure with Fig. 3 a), it can be seen that the system develops more chaotic regions than system with no feedback in the laser. Furthermore, Fig. 4 and the zoom (Fig. 4, right panel), clearly evidence that system II is more chaotic for the whole range of offset phases than the system without feedback in the laser and than system I with the same $J_{1}$.

In Fig. 9 we plot the bifurcation diagram as function of the injected current $J_{0}$ for $J_{1}=0.1 J_{0}$ and two values for the offset phase. This figure is to be compared with Figs. 5 a) and b) for the case without feedback in the laser. For both values of the offset phase chaos starts to develop for a lower value of the pump current $J_{0}$. Fig. 10 shows the bifurcation 

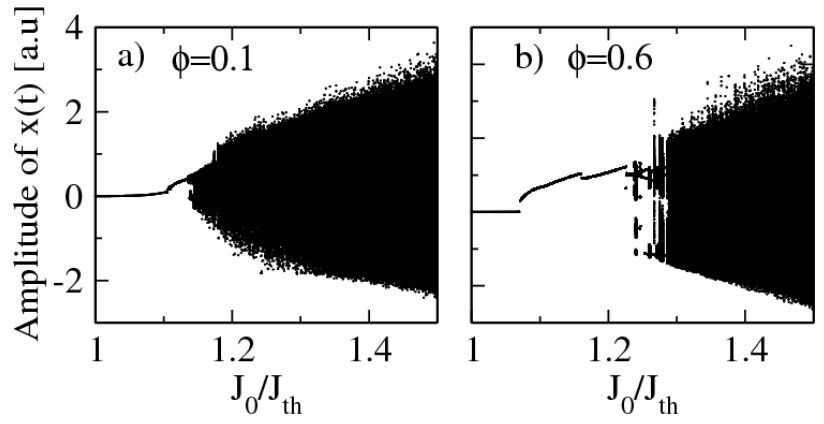

Fig. 9. Bifurcation diagram versus injection current $J_{0}$ for different offset phases $\phi$ considering $J_{1}=0.1 J_{0}$ : a) $\phi=0.1$ and b) $\phi=0.6$.
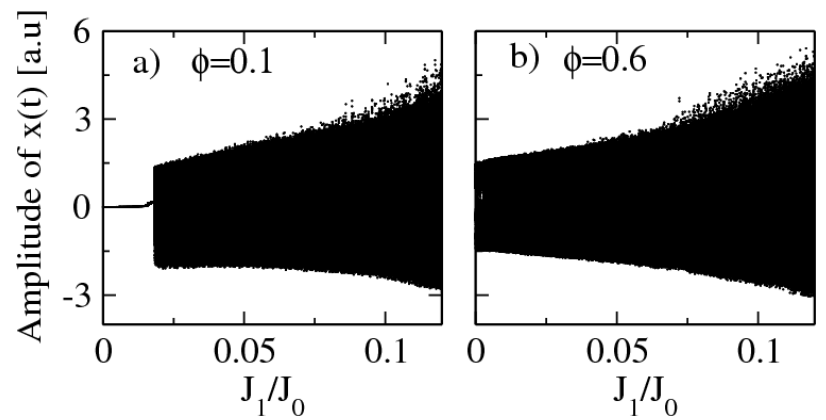

Fig. 10. Bifurcation diagram versus $J_{1}$ for different offset phases $\phi$ considering $J_{0}=1.5 J_{t h}:$ a) $\phi=0.1$ and b) $\phi=0.6$.

diagram as function of the laser feedback strength parameter $J_{1}$ for a fixed $J_{0}=1.5 J_{t h}$. It can be seen the feedback in the laser induces chaos for the offset phase $\phi=0.1$ for which the system is stable without $J_{1}$ and this happens even faster than for system I. An increase in $J_{1}$, for a fixed value of offset phase $\phi=0.6$ leads to a more chaotic behavior as was also the case for system I. As shown in Fig. 7 an increase in $J_{1}$ leads to a rapid decrease of the correlation time. For $J_{1}<0.03 J_{0}$ system I shows a correlation time smaller than system II with the same parameters. As $J_{1}$ increases the correlation time decreases faster for system II. Therefore for $J_{1}>0.03 J_{0}$ system II is more chaotic (has a smaller correlation time) than system I. We have also noticed that the correlation for system II keeps decreasing with $J_{1}$ in the range of parameters we have explored (see the inset plot of Fig. 7).

On the other hand it is also possible to evidence the increase in chaos complexity by plotting the power spectrum of the optical signal transmitted from the emitter to the receiver. The optical output intensity of the MZ is given by $\beta_{1} I(t) \cos ^{2}[x(t)+\phi]$. Figure 11 shows the optical power spectrum. The peak at frequency zero corresponds to the DC component of the optical power output. What matters for chaos codification is the rest of the spectrum beyond the DC peak. For $J_{1}=0$, when no feedback is applied to the laser, (black line) the power spectrum is broad but has some clear peaks signaling that the system is chaotic but the chaos is not fully developed. In the range of $60 \mathrm{GHz}$ the spectrum spreads over $30 \mathrm{~dB}$. For $J_{1}=0.1 J_{0}$ (grey line, red online) the peaks disappear and the spectrum becomes roughly flat. In this case,

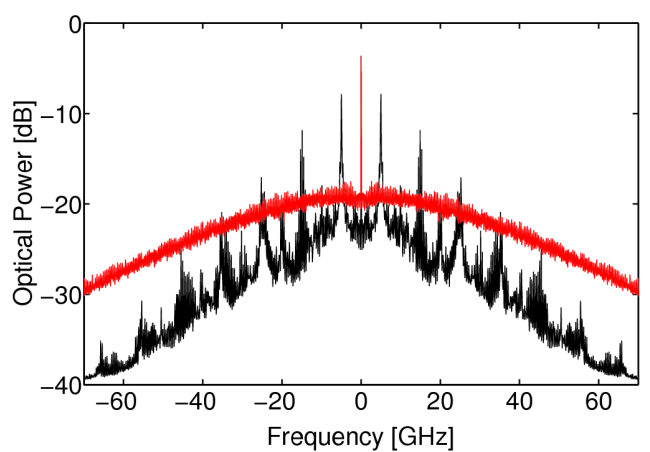

Fig. 11. Optical power spectrum at the output of $\mathrm{MZ}$ considering $J_{0}=$ $1.5 J_{t h}$ and $\phi=0.6: J_{1}=0$ (black line), $J_{1}=0.1 J_{0}$ for system II (grey, red online).

besides the DC peak, $10 \mathrm{~dB}$ are enough to sweep over the $60 \mathrm{GHz}$ range.

\section{RECEIVER SYSTEM AND EFFECTS OF PARAMETER MISMATCH}

\section{A. Receiver system}

For each model, the receiver is built similarly to the emitter. The only difference is that the receiver is fed back by the light only coming from the master instead of itself light. The full emitter-receiver scheme of System I is represented in Fig. 1 c). The feedback in the receiver laser comes from the RF driver output. System II is built similarly but feedback in the receiver laser comes from the $\mathrm{MZ}$ output. Introducing $y(t)=$ $\pi V_{s}(t) /\left(2 V_{\pi}\right)$, the receiver dynamics is given by

$$
\begin{aligned}
& \frac{d I_{s}}{d t}=\left(\mathcal{G}_{s}-\gamma_{s}\right) I_{s}, \\
& \frac{d N_{s}}{d t}=J_{0 s}-\gamma_{e s} N_{s}-\mathcal{G}_{s} I_{s}+F(y), \\
& y+\tau_{s} \frac{d y}{d t}+\frac{1}{\theta_{s}} \int_{t_{0}}^{t} y(s) d s=\beta_{1 s} I_{s}(t-T) \\
& \times \cos ^{2}\left[x\left(t-T_{s}\right)+\phi_{s}\right] .
\end{aligned}
$$

where the subscript " $s$ " denotes the slave. The quality of the synchronization depends on several factors, including the parameter mismatch between master and receiver, the presence of noise fluctuations and the degradation due to fiber propagation effects. The latter has been considered in [18] where the authors showed that compensating the losses by inlining EDFAs every $50 \mathrm{~km}$ and using dispersion shifted fiber, one can minimaze the fiber effects to the very acceptable level. Also, in ref. [19] similar results were found out using standard transmission fibers and then compensating the dispersion using dispersion compensation modules. Here we neglect the effect of noise fluctuations and therefore we focus on the effect of parameter mismatch. We first consider that all the receiver parameters are identical to the master except for the time delay.

Figures 12, show the time trace of the receiver when the two time delays are different. The receiver synchronizes with the master shifted in time at $\Delta T=T_{s}-T$ as can be noticed from Fig. 12 left panel for system I (right panel for 

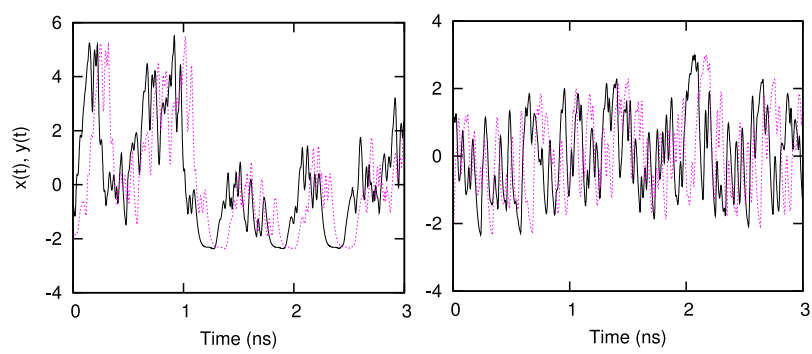

Fig. 12. Emitter (solid line) and receiver (dashed line) time traces for $\Delta T=$ $-0.1 \mathrm{~ns}$ considering left panel: system I and right panel: system II. $J_{0}=$ $1.5 J_{t h}, J_{1}=0.1 J_{0}$ and $\phi=0.6$

system II). Thus mismatches in the delay time produce a trivial effect. The flying time, delays induced by the electrical connections, and the response time of the system (which play exactly the same role as the receivers delay time) just shift the time traces [20]. However, a slight difference on others parameters between the emitter and receiver parameters due to manufacturing mismatches, which are unavoidable, can degrade the synchronization as addressed in the next subsection.

\section{B. Effects of parameter mismatch}

The quality of the synchronization between master and receiver can be characterized by the synchronization error and the cross-correlation. The average synchronization error is given by

$$
\sigma=\sqrt{\frac{\left\langle\varepsilon_{\Delta p}^{2}\right\rangle}{\left\langle x_{p}^{2}\right\rangle}}
$$

where $\varepsilon_{\Delta p}=y_{p^{\prime}}(t)-x_{p}(t)$. This is an indicator of the minimum modulation amplitude below which the encoded message cannot be recovered. The subindexes $p$ and $p^{\prime}$ in the master and slave time traces reflect the fact that the master and slave systems may operate with different parameter values. The cross-correlation

$$
\Gamma_{x y}\left(t^{\prime}\right)=\frac{\left\langle\left[x_{p}(t)-\left\langle x_{p}(t)\right\rangle\right]\left[y_{p^{\prime}}\left(t+t^{\prime}\right)-\left\langle y_{p^{\prime}}(t)\right\rangle\right]\right\rangle}{\sqrt{\left\langle\left|x_{p}(t)-\left\langle x_{p}(t)\right\rangle\right|^{2}\right\rangle\left\langle\left|y_{p^{\prime}}(t)-\left\langle y_{p^{\prime}}(t)\right\rangle\right|^{2}\right\rangle}},
$$

is a qualitative indicator of the topological distortion of slave trajectory. In the following, we analyze the influence of mismatch in each parameter individually. It is worth noting that for $\Delta T=0$, the correlation is maximum for $t^{\prime}=0$.

Figure 13 shows the synchronization error (left panels) and the cross-correlation (right panels) for different mismatches in the laser parameters. For a mistmach in $N_{0}$ and $\gamma_{e}$, the synchronization error grows basically linearly with the mismatch, and the cross-correlation function shows a parabolic shape. Positive and negative mismatches have similar effects. The degradation of the synchronization with the mismatch is stronger for system II (+) as compared with system I ( $($ ) or the system without laser feedback (solid line). The mismatch in $\gamma$ is the one for which both system I and system II are more sensitive. The effect of this mismatch is very asymmetric
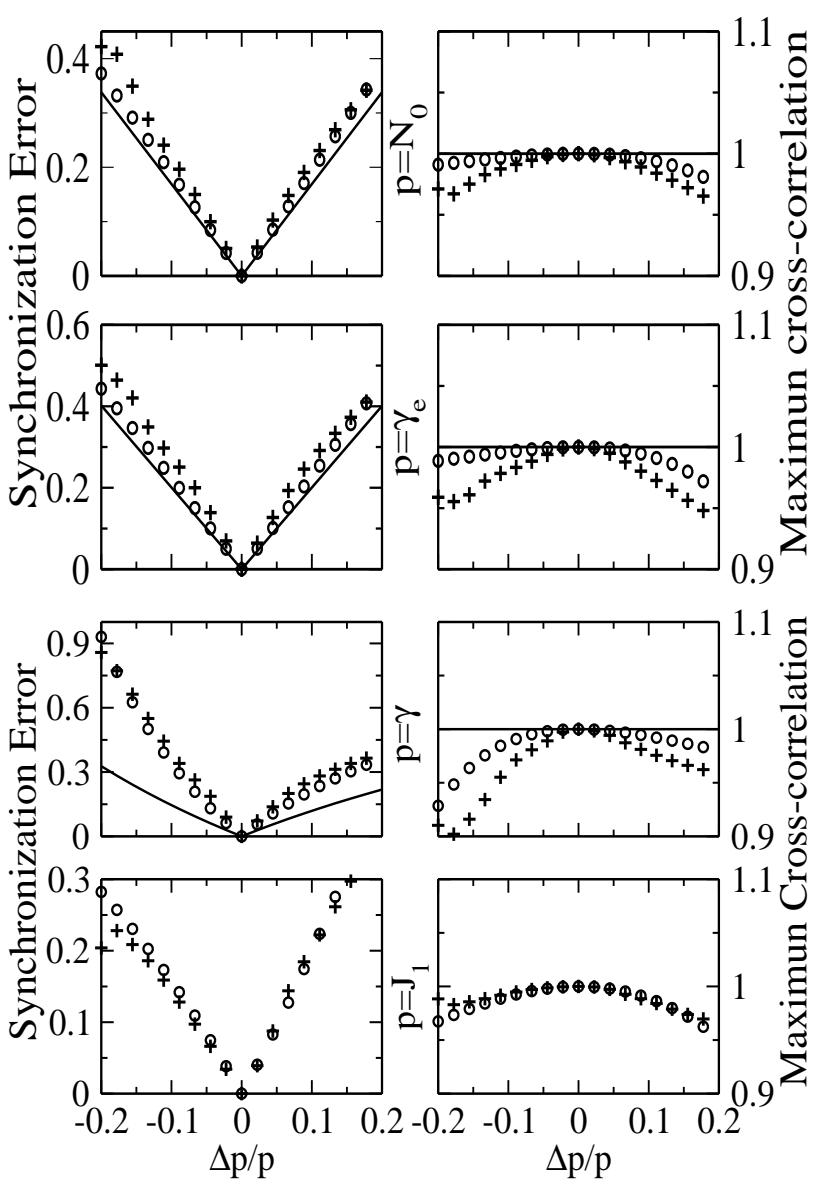

Fig. 13. Average synchronization error (left panels) and maximum crosscorrelation (right panels) given by Eqs. 16 and 17, respectively, as function of the laser parameter mismatch $\left(\Delta p=p^{\prime}-p\right)$ in $N_{0}, \gamma_{e}, \gamma$ and $J_{1}$ (from top to bottom). The solid line corresponds to $J_{1}=0$ (no feedback in the laser), (o) to system I with $J_{1}=0.1 J_{0}$ and (+) to system II also with $J_{1}=0.1 J_{0}$.

being more pronounced for negative $\left(\gamma_{s}>\gamma\right)$ than for positive mistmatch. This asymmetry is already present when the laser operates in $\mathrm{CW}$ (solid line) and is magnified by the feedback in the laser. Furthermore, System II is slightly more sensitive to the mismatch than System I, the difference being more relevant for the cross-correlation. The mismatch in $J_{1}$ induces a synchronization error that grows asymmetrically with the mismatch. For positive mismatch $\left(J_{1 s}<J_{1}\right)$ the degradation grows linearly and faster than for negative mismatch (where the synchronization error grows sublinearly). The cross-correlation also shows this asymmetry.

As for the other parameters, the effect of mismatch in the slow cut-off time $\theta$ is typically negligible in this kind of systems [20], so we focus on the mismatch in the offset phase $\phi$ and the fast cut-off time $\tau$. Results are plotted in Fig. 14. The synchronization quality is very sensitive to the offset phase mismatch. When the laser operates in CW (solid line) by changing the offset phase we move from perfect synchronization (for zero or $\pi$ mismatch) to perfect antisynchronization (for $\pm \pi / 2$ mismatch) in which $y(t)=-x(t)$ $\left(\sigma=2\right.$ and $\left.\Gamma_{x y}=-1\right)$. When the feedback is added to the laser, perfect synchronization is still present for zero and $\pi$ 


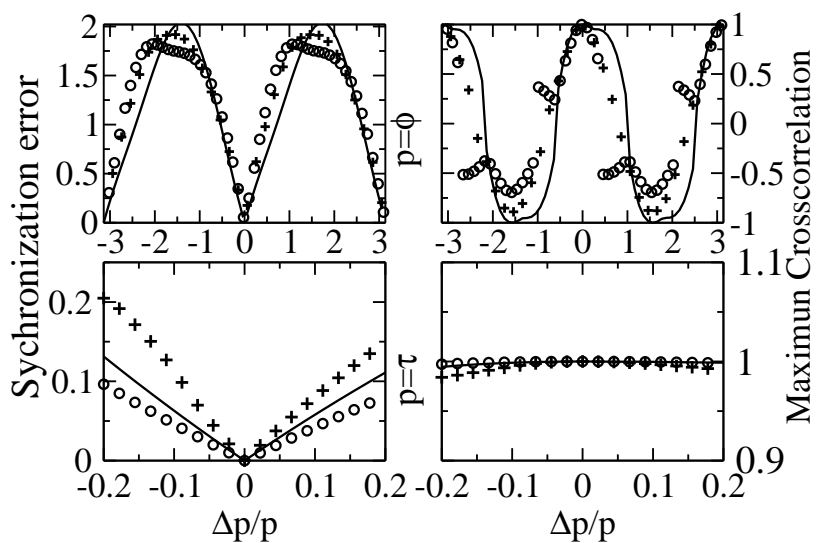

Fig. 14. Average synchronization error (left panels) and maximum crosscorrelation (right panels) given by Eqs. 16 and 17, respectively, as function of the filter loop parameter mismatch in $\phi$ (top row) and $\tau$ (bottom row). The solid line corresponds to $J_{1}=0$ (no feedback in the laser), (o) to System I with $J_{1}=0.1 J_{0}$ and (+) to System II also with $J_{1}=0.1 J_{0}$.

mismatch but the perfect anti-synchronization is no longer obtained. System II presents a better anti-synchronization than system I. The fast cut-off time $\tau$ mismatch plays a very different role in system I and II. In system I the effect of mismatch in $\tau$ is slightly less important than that obtained when the laser operates in $\mathrm{CW}$ while the degradation of the synchronization for system I grows faster as the mismatch increases. Nevertheless the effect of $\tau$-mismatch is always less important than the other mismatches discussed before.

\section{ENCODED/DECODED MESSAGE}

In all optical chaos communication systems, the message to be encrypted should be small compared to the amplitude of chaotic carrier to ensure the secrecy and to avoid large distortions of the transmitter output which could prevent the receiver to synchronize with the emitter. When the message is encoded, the system performances can be affected in different ways (dynamics, synchronization and communication performance) depending on the encoding scheme [21]. Numerical simulations have shown that the additive chaos masking (ACM) scheme can increase the complexity of the chaotic waveforms maintaining at the same time the synchronization quality before and after a message is encoded [22].

In the scheme shown in Fig. $1 \mathrm{c}$ ), we encode the message using the ACM technique. We assume the message to be encoded in the chaotic system as a sequence of a non-returnto-zero (NRZ) pseudorandom digital bits at $1.0 \mathrm{Gbit} / \mathrm{s}$ rate. The message is added to the emitter carrier by using another semiconductor laser with a similar wavelength. In Fig. $1 \mathrm{c}$ ), the output of the laser diode containing message is $p_{1} m(t)$ where $m(t)$ is 1 when an optical power $p_{1}$ is transmitted and 0 when no optical power is transmitted. The mixing is performed through an all-optical $2 \times 2$ fiber coupler which is also used to send the output to the receiver.

When the message is embedded within the chaos, Eq. (1)
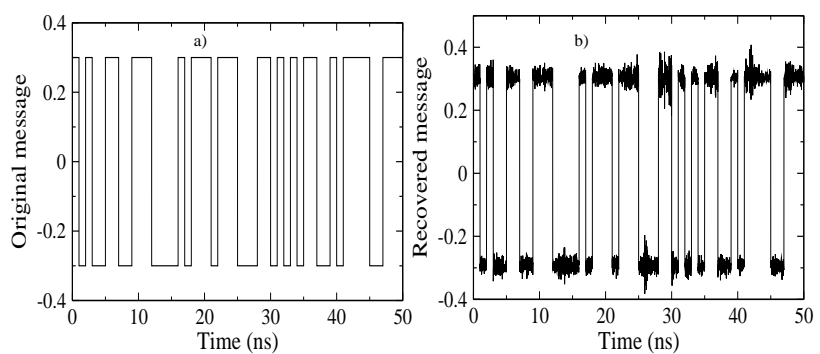

Fig. 15. a) original message and b) recovered message, performed with model II considering $-0.5 \%$ and $0.1 \%$ mismatch in $\tau$ and $\gamma$ respectively. We have taken $J_{0}=1.5 J_{t h}, J_{1}=0.1 J_{0}$ and $\phi=0.6$

becomes

$$
\begin{aligned}
x+\tau \frac{d x}{d t}+\frac{1}{\theta} w= & \beta_{1} I(t-T) \cos ^{2}[x(t-T)+\phi] \\
& +\alpha m(t-T),
\end{aligned}
$$

where $\alpha=\pi P_{1} / 2 V_{\pi}$ is the amplitude of the message. Here we use $\alpha=0.3$ which is much smaller than the average gain $\left\langle\beta_{1} I\right\rangle \approx 3.08$.

At the input of the receiver, the transmitted signal is split in two parts, one is to drive the slave system and the other is directly detected by the photodiode $P D_{+}$. Taking into account the message, the slave system dynamics is ruled by

$$
\begin{aligned}
y+\tau \frac{d y}{d t}+\frac{1}{\theta} w_{s}= & \beta_{1} I_{s}(t-T) \cos ^{2}[x(t-T)+\phi] \\
& +\alpha m(t-T) .
\end{aligned}
$$

The output of the slave system is detected by photodiode $P D_{-}$. Subtracting $P D_{-}$from $P D_{+}$one gets

$$
\begin{aligned}
S_{c}(t)= & k^{\prime}\left\{\beta_{1} I \cos ^{2}[x(t)+\phi]+\alpha m(t)\right\} \\
& -k \beta_{1} I_{s} \cos ^{2}\left[y(t)+\phi_{s}\right],
\end{aligned}
$$

where $k$ and $k^{\prime}$ are factors accounting for the $P D_{-}$and $P D_{+}$ sensitivities and fiber couplers, respectively. If the receiver and emitter synchronize perfectly, $x(t)=y(t), I(t)=I_{s}(t)$, and taking $k=k^{\prime}, \phi_{s}=\phi$, we have from Eq. (20)

$$
S_{c}(t)=k \alpha m(t) .
$$

For $k=1$ and $\alpha=0.3$, the retrieved message performed considering $-0.5 \%$ and $0.1 \%$ mismatch in $\tau$ and $\gamma$ respectively, is shown in Fig. 15 using system II. As it can be seen from the figure, the message can be well recovered.

\section{CONCLUSIONS}

In this paper, we have studied two electro-optical delay feedback systems pumped by a semiconductor laser subject to feedback. In particular we have introduced two new different schemes in which this can be implemented.

The additional feedback in the laser makes the semiconductor laser to operate in a chaotic regime instead of CW. As a consequence the system becomes more chaotic as shown by a shorter autocorrelation time. Furthermore the additional 
feedback induces chaos for parameters values in which the system was not chaotic. These effects are already clearly observed when the feedback in the semiconductor lasers is only $10 \%$ of the DC pump current. Therefore this is a useful mechanism to generate broader bandwidth chaos.

Despite the systems are more chaotic, high-quality synchronization is still possible when the mismatch in parameters is small allowing for message transmission. The additional parameters to be tuned, in order to achieve synchronization, increase the difficulties of an eventual eavesdropper to decode the message without the adequate receiver system.

\section{REFERENCES}

[1] J. P. Goedgebuer, L. Larger, and H. Porte, Optical cryptosystem based on synchronization of hyperchaos generated by a delayed feedback tunable laser diode, Phys. Rev. Lett. vol. 80, pp. 2249-2252, 1998.

[2] L. Larger, J. P. Goedgebuer, and F. Delorme, Optical encryption system using hyperchaos generated by an optoelectronic wavelength oscillator, Phys. Rev. E vol. 57, pp. 6618-6624, 1998.

[3] L.M. Pecora and T.L. Carroll, Synchronization in chaotic systems, Phys. Rev. Lett. vol. 64, pp. 821-824, 1990.

[4] K. M. Cuomo and A. V. Oppenheim, Circuit implementation of synchronized chaos with applications to communications, Phys. Rev. Lett. vol. 71, pp. 65-68, 1993.

[5] P. Colet and R. Roy, Digital Communication with Synchronized Chaotic Lasers, Opt. Lett. vol. 19, pp. 2056-2058, 1994.

[6] C.R. Mirasso, P. Colet, P. Garcia-Fernandez, Synchronization of Chaotic Semiconductor Lasers: Application to Encoded Communications, IEEE Photon Technol. Lett. vol. 8, pp. 299-301, 1996.

[7] V. Annovazzi-Lodi, S. Donati, and A. Scir, Synchronization of chaotic injected-laser systems and its application to optical cryptography, IEEE J. Quantum Electron. vol. 32, pp. 953-959, 1996.

[8] G.D. Van Wiggeren and R. Roy, Communications with chaotic lasers, Science vol. 279, pp. 1198-1200, 1998.

[9] A. Argyris, D. Syvridis, L. Larger, V. Annovazzi-Lodi, P. Colet, I. Fischer, J. Garcia-Ojalvo, C.R. Mirasso, L. Pesquera, K.A. Shore, Chaosbased communications at high bit rates using commercial fibre-optic links, Nature vol. 438, pp. 343-346, 2005.

[10] Feature Section on Optical Chaos and Applications to Cryptography, edited by S. Donati and C.R. Mirasso, IEEE J. Quantum Electron. vol. 38, pp. 1138-1184, 2002.

[11] J. Goedgebuer, L. Larger and H. Porte, Chaos in wavelength with a feedback tunable laser diode, Phys. Rev. E vol. 57, pp. 2795-2798, 1998.

[12] R. Vicente, J. Dauden, P. Colet, R. Toral, Analysis and Characterization of the Hyperchaos Generated by a Semiconductor Laser Subject to a Delayed Feedback Loop , IEEE J. Quantum Electron. vol. 41, pp. 541548,2005

[13] V. S. Udaltsov, L. Larger, J. P. Goedgebuer, A. Locquet and D. S. Citrin Time delay identification in chaotic cryptosystems ruled by delaydifferential equations, J. Opt. Technol. vol. 72, pp. 373-377, 2005.

[14] G. P. Agrawal and N. K. Dutta, Long Waves Semiconductor Lasers, New York: Nostrand Reinhold, 1986

[15] V. Z. Tronciu, C. R. Mirasso and P. Colet, Chaos-based communications using semiconductor lasers subject to feedback from an integrated double cavity, J. Phys. B: At. Mol. Opt. Phys. vol. 41, pp. 155401/1$155401 / 8,2008$.

[16] G. P. Agrawal, Noise in Semiconductor Lasers and its impact in optical communication systems, In proc. SPIE. vol. 1376, R. Roy, Ed., pp. 224$235,1990$.

[17] K. Engelborghs, T. Luzyanina, G. Samacy, Report TW 330, 2001.

[18] A. Bogris, D. Kanakidis, A. Argyris, and D. Syvridis, Performance characterization of a closed-loop chaotic communication system including fiber transmission in dispersion shifted fibers, IEEE J. Quantum Electron., vol. 40, pp. 1326-1336, 2004.

[19] D. Kanakidis, A. Bogris, A. Argyris, and D. Syvridis, Numerical investigation of fiber transmission of a chaotic encrypted mes- sage using dispersion compensation schemes, IEEE J. Light-wave Technol., vol. 22, pp. 2256-2263, 2004.

[20] Y. Chembo Kouomou, P. Colet, N. Gastaud and L. Larger, Effect of parameter mismatch on the synchronization of semiconductor lasers with electrooptical feedback, Phys. Rev. E vol. 69, pp. 056226/1056226/15, 2004.
[21] Shuo Tang and Jia-Ming Liu, Effects of message encoding and decoding on synchronized chaotic optical communications, IEEE J. Quantum Electron., Vol. 39, pp. 1468-1475, 2003.

[22] J. G. Ford , M. G. Leonard , J. Swiss and G. C. Gainer, INSULDUR, Phys. Rev. A, vol. 65, pp. 033804/1-033804/16, 2002.

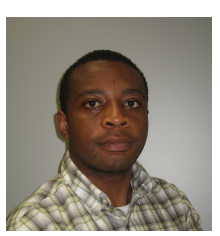

Romain Modeste Nguimdo was born in FongoTongo, Cameroon, on March 15, 1981. He received the M.Sc. degree in theoretical physics from the Universities of Dschang and Yaoundé I, Cameroon. He is currently working toward the Ph.D. degree from the Instituto de Física Interdisciplinar y Sistemas Complejos, IFISC (CSIC-UIB), Palma de Mallorca, Spain. His research interest involves nonlinear dynamics, optical chaos cryptography.

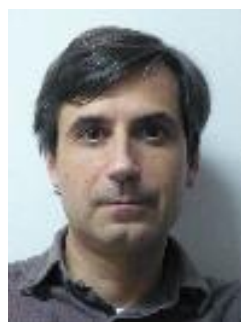

Pere Colet was born in Vilafranca del Penedes, Barcelona, Spain, on April 21, 1964. He received the M.Sc. degree in physics in 1987 from the Universitat de Barcelona, and the Ph.D. degree in physics in 1991 from the Universitat de les Illes Balears, Palma de Mallorca, Spain. In 1991, he became a Teaching Assistant at the Departament de Fisica, Universitat de les Illes Balears. From September 1991 to February 1993 and from April to September 1994, he was a Postdoctoral Fulbright Fellow at the School of Physics, Georgia Institute of Technology, Atlanta. In October 1994, he joined the Departament de Fisica, Universitat de les Illes Balears. Since May 1995, he has held a permanent research position at the Spanish Consejo Superior de Investigaciones Científicas. His present position is Reseach Professor at IFISC in Palma de Mallorca. $\mathrm{He}$ has co-autored over 90 papers in SCI journals as well as other 30 scientific publications. His research interests include fluctuations and nonlinear dynamics of semiconductor lasers, synchronization of chaotic lasers and encoded communications, synchronization of coupled nonlinear oscillators, pattern formation and quantum fluctuations in nonlinear optical cavities and dynamics of localized structures.

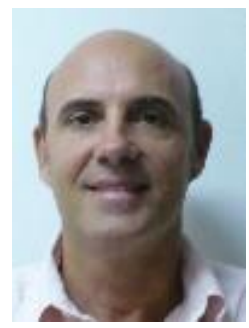

Claudio R. Mirasso was born in Buenos Aires, Argentina, in 1960. He received the M.Sc. and Ph.D. degrees in physics from the Universidad Nacional de La Plata, Buenos Aires, Argentina, in 1984 and 1989, respectively. After several Postdoctoral positions in Spain and the Netherlands, he became an Associate Professor with the Physics Department, Universiteit de les Illes Bolears (UIB), in 1996. He has authored or coauthored over 140 publications including about 110 journal papers. His research interests include instabilities in semiconductor lasers, synchronization, and control of chaotic semiconductor lasers, dynamics and applications of delayed coupled semiconductor lasers, and applications of nonlinear dynamics. 